\title{
A Case Series Report: Prelingually Deaf Cochlear Implant Users and Factors Associated with Outcomes
}

\author{
Ming Zhang ${ }^{1,2,3, *}$, Emily Hill ${ }^{1}$, Adrianne Boyd ${ }^{1}$ \\ ${ }^{1}$ Speech Pathology and Audiology, University of Alberta - Faculty of Rehabilitation Medicine, Edmonton, AB T6G2G4, Canada \\ ${ }^{2}$ Audiology, Alberta Health Services - Glenrose Rehabilitation Hospital, Edmonton, AB T5G 0B7, Canada \\ ${ }^{3}$ Surgery - Otolaryngology, University of Alberta - Faculty of Medicine and Dentistry, Edmonton, AB T6G 2B7, Canada \\ *Corresponding Author: ming.zhang@ualberta.ca
}

Copyright $(2014$ Horizon Research Publishing All rights reserved.

\begin{abstract}
Approximately 219,000 people worldwide have received cochlear implants (CI) as of 2010. This retrospective study uniquely investigated three important components together including pre-lingual CI recipients (the most difficult-to-treat CI population), speech recognition before and after CI, and factors that may be associated with positive or negative speech recognition outcomes. Eight cases of pre-lingual CI users were selected, including four subjects with relatively better scores and four subjects with relatively poor scores. To compare these two groups, eight factors were investigated: gender; etiology; age of implantation; type of implant device; communication mode (oral, speech reading, or sign); patient compliance (attending scheduled clinic follow-up); family/environmental influence; and frequency using the CI device. Although the finding from this investigation is not statistically conclusive like other similar studies, it appears that some factors, such as patient compliance, oral communication, family environment, and/or the frequency using the $\mathrm{CI}$ device, may contribute to positive speech recognition outcomes.
\end{abstract}

Keywords Cochlear Implant, Prelingual Deafness, Outcome, CI Recipients

\section{Introduction}

In this technological day and age, the advancements in cochlear implant (CI) technology and procedures for individuals with profound sensorineural hearing loss are constantly improving. Research shows that earlier implantation in children leads to better speech and language development outcomes[1] and, specifically, that implantation before two years of age is better than later[2]. Thus, if "earlier is better," individuals who are prelingually deafened, or, those whose hearing loss occurred before two years of age[3], may demonstrate less than ideal performance on clinical measures if he/she were not implanted until after the critical period of speech and language acquisition. This is because, without hearing, a person may experience difficulty learning and understanding spoken language[4]. Furthermore, CI users' speech and language skills are often not as refined as individuals with normal hearing[5], especially in prelingual CI users[6]. In this paper, an individual deafened before two years of age but implanted post-critical speech/language development shall be termed a "prelingual CI user." On the other hand, a myriad of variables and factors (age at implantation, mode of communication, rehabilitation services, family expectations, motivation, etc.) have been mentioned that may affect the performance and outcomes (speech recognition scores) of CI users[7]. Therefore, our objective was to investigate eight prelingual CI users by reviewing eight potential affecting factors, which included gender, etiology, age of implantation, type of device, communication (oral, speech reading, or sign), patient compliance (attendance for scheduled medical appointments), and family and environment. Our research question asked whether or not these eight factors could affect the outcomes (speech recognition scores) of prelingual users; we hypothesized that the outcomes of prelingual users may be affected by most of these eight factors.

\section{Materials and Methods}

\subsection{Design}

For this case series study, two steps were used in data collection. First, the data of the cases were retrospectively collected previously from cases' medical files at the Glenrose Rehabilitation Hospital. The collected data were prepared as format on a series of notes. Second, from the data available on these notes, the cases for this report were chosen based on whether their records provided pre and post-implantation speech recognition scores. The scores were analyzed, and then the subjects were subsequently sorted into "good" or "poor" outcome groups. To ensure consistency of outcome measurement, subjects were included if they had records of speech recognition scores on 
the Central Institute for the Deaf (CID) - Everyday Sentences test or the Hearing In Noise Test (HINT). CID and HINT were not compared with each other but compared separately with each self between pre- and post-implant scores within subjects. By using calibrated and monitored speech materials, the CID (live voice) and HINT (playback voice) were presented at 60-70 dB SPL. These tests measure an individual's ability to correctly recognize words in sentences spoken out in an appropriate loudness, expressed as a percentage of correctly recognized words. With the eight subjects eligible for this study, factors were extracted from the notes available from the medical charts pertaining to gender, etiology, age of implantation, type of device, method(s) of communication (e.g. oral, speech reading, sign), patient compliance (attendance for scheduled medical appointments), and family and environment.

\subsection{Sample}

The subjects in this study were all prelingually deaf people who had received a CI unilaterally. Inclusion criteria included the availability of both pre and post assessment measures around average one year after CI surgery, prelingual deafness with severe to profound hearing loss, and CI surgery after two years of age. As data was drawn from medical files, certain criteria were not always present for each subject.

\subsection{Measures}

This paper looked at CI users' speech recognition scores and addressed the influence of eight possible factors (e.g. gender, age, etc.) that may have contributed to either poor or successful speech recognition outcomes. These factors are important because they have been studied by a number of investigators in the past as reviewed below.

\section{Series Report I: Eight Cases}

Case 1. The subject was a fifteen year of female who presented with bilateral profound sensorineural hearing loss with a history of DiGeorge Syndrome. She had experienced pressure equalization tubes placement due to middle ear problem, although this might not be relevant to her sensorineural hearing loss. At approximately eight years old, she required a bilateral mastoidectomy due to her ear infections. She also received hearing aids and had worn them since then. Shortly after, she received a BAHA device as well. Case 1 used these assistive devices until age ten years, when she could no longer hear using the BAHA. She had received closure surgery at eleven years old. When she was eleven years old, due to failure to using assistive device for her hearing, a Nucleus Cochlear Freedom Contour CI was implanted in her right ear. All electrodes were activated. It was indicated in her file that she used her CI all waking hours and her speech had improved significantly, although she was still severely delayed in oral language development. It was also reported that she was able to use the telephone. However, she has difficulty hearing in background noise. The data also indicated that case 1 was an auditory oral communicator, and that she reported being happy with her CI. In addition, she required fewer programming changes compared to other CI users. It was noted that she attended medical appointments according to her schedule in regards to her CI. This subject had been assessed both pre and post-surgery for speech recognition using the HINT in quiet. Her scores were as follows, $0 \%$ pre implant and $100 \%$ post implant.

Case 2. The subject was a thirty-four year old female who was implanted in her left ear at age twenty-nine. She presented with profound sensorineural hearing loss bilaterally and did not have any auditory brainstem response. She had been fitted with hearing aids at approximately three years old. She had once worn a hearing aid in her left ear, but with very limited benefit. She also described constant tinnitus in her left ear, but did not report any serious balance problems. She then discontinued wearing the device, as she did not think she could benefit from using it. In addition, she did not use a hearing aid in her right ear because she did not report any benefit from wearing the device. She completely discontinued using the hearing aid five years before cochlear implant surgery. At the age of twenty-nine, she received a Nucleus Cochlear Freedom Contour CI. All electrodes were activated. Since her surgery, the subject indicated that she had begun to rely less on speech reading and more on her auditory abilities. She started using the telephone in a limited fashion and stated that she was beginning to enjoy music. Continued benefit from the CI was noted for the subject four years after the implantation surgery as noted in her medical file. She had been assessed both pre- and one year post-surgery using the CID everyday sentence test (auditory only) receiving scores of $0 \%$ and $67 \%$ respectively.

Case 3. The subject was a twenty-six year old female who presented with profound sensorineural hearing loss in her right ear and severe-profound sensorineural hearing loss in her left before implantation. The underlying etiology and the progression of her hearing loss were unknown. Although irrelevant to her sensorineural hearing loss, it was stated in her medical file that she had had a history of middle ear problems, including otitis media. As well, she had undergone a middle ear surgery in her right ear and received pressure equalization tubes. The CI surgery had been re-scheduled several times due to aforementioned middle ear difficulties. At the age of nineteen years, she received an Advanced Bionics device 90k CI in her right ear. All electrodes were activated. It was noted in the file that one year post-surgery, she had excellent hearing sensitivity and good speech discrimination with her CI; however, she continued to have problems with middle ear fluid and had severely reduced tympanic compliance bilaterally. The subject had been assessed both pre and one year post-surgery using the HINT in quiet. Her pre implantation score was $0 \%$ and her post implantation score was $30 \%$. It was reported that the subject attended all scheduled medical appointments regarding her 
CI.

Case 4. The subject was a twelve year old female. Before implantation, she presented with profound sensorineural hearing loss and an unknown etiology. At the age of four years old, she was implanted in her left ear with a Nucleus 24 Contours with Freedom speech processor. All electrodes were activated. It was noted in the file that the subject communicated orally, as well as through sign language and speech reading. She had been making progress with her CI; however, she often complained that it was too soft and that she struggled to use it for work. The issue of her CI mapping has been mentioned in her file for her CI to work. It was also indicated that the subject wore her CI but used her FM system less often. She was tested both pre and post implantation. Her pre implantation score of Glendonald Auditory Screening Procedure (GASP) sentences was $0 \%$. Her post 3-year score of CID everyday sentences was $22 \%$. GASP test is easier, and so CID score will not be higher than GASP score.

Case 5. The subject was a twenty-eight year old male who had profound sensorineural hearing loss bilaterally. It was speculated in his medical file that his hearing loss could have been due to his mother having German measles during her pregnancy. He had worn bilateral BTE hearing aids until he was sixteen years old when he discontinued using his left hearing aid. The subject used both oral and manual communication before implantation. He was implanted in his right ear with a CI 512 Nucleus at the age of approximately twenty-seven. All electrodes were activated. It was stated in his file that he enjoyed music although he desired an increased volume. Furthermore, he found it difficult to follow conversations. His CI was reprogrammed one year after the implantation and he reported a significant improvement in the area of clarity of speech. The subject was assessed pre and post-surgery using the HINT in quiet. His pre score was $44 \%$ and his post score was $48 \%$.

Case 6. The subject was a twenty-two year old male who presented with a diagnosis of Waardenburg's syndrome and profound sensorineural hearing loss bilaterally. It was discussed in his file that he used speech reading and note pads as a form of communication pre-surgery. He had used Signed Exact English (SEE) in high school but did not use it after that. The subject reported that he had always worn hearing aids and that his hearing had not changed a lot throughout his life. He was implanted with a Freedom Contour advance in his right ear at the age of eighteen. All electrodes were activated but basal 3 that may cause vestibular and facial stimulation. A year post surgery, he stated that his primary benefit from the CI was improved sensitivity and that he was able to hear softer sounds better than he had with his hearing aids. Medical reports indicated that a year and a half after implantation, his speech intelligibility had improved since his last appointment, although his speech was not $100 \%$ intelligible. Two years post-surgery, it was noted that the subject did not wear his device during the day due to his work environment; however, he wore both his CI and his hearing aid (in left ear) in the evenings. The subject had been assessed both pre and post-surgery with the CID everyday sentence test (auditory only) and received a score of $1 \%$ and $2 \%$ respectively.

Case 7 . The subject was a forty-four year old female who presented with profound sensorineural hearing loss bilaterally. The etiology causing her deafness was unknown. She had been wearing hearing aids since she was two years old, but had made minimal gains over her performance with her hearing aids. She had had no history of balance problems or ear surgeries. She did have a history of arthritis, but it was irrelevant to her hearing loss. The subject was implanted at age forty-one with a Freedom Contour Advance CI. All electrodes were activated. It was indicated in her file that she had been slow to adjust to the CI device. The subject communicated orally and had learned some sign language. The file reported that the subject had excellent family support. Two years post implantation, she continued to show problems with speech recognition. The subject had been assessed using the CID everyday sentence test (auditory only) both pre and post implantation. Her scores were $9 \%$ initially and $4 \%$ after one year of surgery. She was tested again two years post-surgery and received a score of $8 \%$.

Case 8 . The subject was a nineteen year old female with bilaterally severe-profound sensorineural hearing loss (etiology unknown). She had worn hearing aids on the right side at all times before her CI. At ten years of age, her right ear was implanted with a CII Bionic 90k with Harmony speech processor. All electrodes were activated. After implantation, she had reported feelings of dizziness and off-balance. One year post-surgery, she reported limited benefit from her CI and would only used it approximately three hours per day; however, she was beginning to wear it more often. Therefore, she was beginning to get more benefits from using the CI. Two and a half years later, she reported that she was beginning to use the telephone and could hear environmental noise. As well, she could sometimes understand people talking without having to look at them, and could also hear people call her name. The CID everyday sentence test (auditory only) was given both pre and post implantation. Her results indicated that the score was low after implantation ( $2 \%$ "unable to correctly identify any words in sentences with open set CID sentences, without visual cues"). 
Table 1. Summary of series cases*

\begin{tabular}{|c|c|c|c|c|c|c|c|c|c|c|}
\hline Case \# & $\begin{array}{c}\text { Gen- } \\
\text { der }\end{array}$ & Age & \begin{tabular}{|c|}
$\begin{array}{c}\text { Age at } \\
\text { implan-ta } \\
\text { tion }\end{array}$ \\
\end{tabular} & Hearing Loss & \begin{tabular}{c|} 
Ear \\
impl-a \\
nted
\end{tabular} & $\begin{array}{l}\text { Speech Recognition } \\
\text { Score (post-pre**) }\end{array}$ & $\begin{array}{c}\text { Type of } \\
\text { Communica- } \\
\text { tion used } \\
\end{array}$ & $\begin{array}{c}\text { Etiology of } \\
\text { Hearing Loss }\end{array}$ & Type of Device & $\begin{array}{l}\text { Comp- } \\
\text { liance }\end{array}$ \\
\hline 1 & $\mathrm{~F}$ & 15 & 11 & $\begin{array}{c}\text { profound SNHL } \\
\text { bilaterally }\end{array}$ & right & $100 \%$ HINT & Oral & $\begin{array}{l}\text { DiGeorge } \\
\text { Syndrome }\end{array}$ & Nucleus Freedom Contour & $\begin{array}{c}\text { Went to all } \\
\text { appoint-m } \\
\text { ents }\end{array}$ \\
\hline 2 & $\mathrm{~F}$ & 33 & 29 & $\begin{array}{c}\text { profound SNHL } \\
\text { bilaterally }\end{array}$ & left & $67 \% \mathrm{CID}$ & $\begin{array}{l}\text { Oral and speech } \\
\text { reading }\end{array}$ & $\begin{array}{l}\text { Mother's } \\
\text { Rubella }\end{array}$ & Nucleus Freedom Contour & \\
\hline 3 & $\mathrm{~F}$ & 25 & 19 & \begin{tabular}{|c|} 
severe-profound \\
SNHL \\
bilaterally \\
\end{tabular} & right & $30 \% \mathrm{CID}$ & Unknown & Unknown & Bionics 90k & \begin{tabular}{|c|}
$\begin{array}{c}\text { Went to all } \\
\text { appoint-m } \\
\text { ents }\end{array}$ \\
\end{tabular} \\
\hline 4 & $\mathrm{~F}$ & 12 & 4 & profound SNHL & left & $22 \% \mathrm{CID}$ & $\begin{array}{l}\text { Oral, sign and } \\
\text { speech reading }\end{array}$ & Unknown & Nucleus Freedom N24 & \\
\hline Mean & & 21 & 16 & & & Range: $22-100 \%$ & & & & \\
\hline 5 & M & 28 & 27 & $\begin{array}{c}\text { profound SNHL } \\
\text { bilaterally }\end{array} \mid$ & Right & 4\% HINT & Unknown & $\begin{array}{c}\text { Maternal } \\
\text { German } \\
\text { Measles }\end{array}$ & Nucleus 512 & \\
\hline 6 & M & 21 & 18 & $\begin{array}{c}\text { profound SNHL } \\
\text { bilaterally }\end{array}$ & Right & $1 \% \mathrm{CID}$ & $\begin{array}{c}\text { Speech reading and } \\
\text { writing }\end{array}$ & $\begin{array}{c}\text { Waardenburg } \\
\text {,s }\end{array}$ & Nucleus Freedom Contour & \\
\hline 7 & $\mathrm{~F}$ & 44 & 41 & $\begin{array}{c}\text { profound SNHL } \\
\text { bilaterally }\end{array}$ & Right & $4 \% \mathrm{CID}$ & Oral and sign & Unknown & Nucleus Freedom Contour & \\
\hline 8 & $\mathrm{~F}$ & 19 & 10 & $\begin{array}{c}\text { profound SNHL } \\
\text { bilaterally }\end{array}$ & Right & $2 \% \mathrm{CID}$ & $\begin{array}{c}\text { Oral and speech } \\
\text { reading }\end{array}$ & Unknown & Bionics 90k & \\
\hline Mean & & 28 & 24 & & & Range: $1-4 \%$ & & & & \\
\hline
\end{tabular}

* This table briefly summarizes the major information of the series cases described above.

** Post-pre is the comparison of post with pre implantation aided scores.

\section{Series Report II: Eight Factors}

Presenting the data in one case after another (e.g., in the section above) is a conventional approach in the literature. Presenting the data in one factor after another, as in this section, is an exceptional approach in which the effect of each factor can be presented across all cases (i.e., patients). Such presentation not only can summarize eight factors (e.g., gender, device type, oral communication, compliance, family, environment, etc.), but also may demonstrate those factors which may have potentially positive effect on treatment outcome.

In this report, three factors show a trend towards positive outcomes, although a statistical goal to show significance (i.e., $\mathrm{p}<0.05$ ) cannot be achieved using Levene's test on a small-paired sample size. Additionally, based on previous studies[6], due to the nature of such study with an extensive variation and lack of uniform data, generally the data would not be statistically significant.

These three factors, which may be potentially positive and lead good performance, are as follows: oral communication; patient compliance; and family and environment. As this section is still the presentation of series report, discussion is not included as it is in section "5. Discussion").

\subsection{Gender}

In the current study, retrospective data was collected for six female and two male prelingual CI users (3:1). Only female subjects improved more than $20 \%$ on their speech recognition scores (comparing pre versus post results); however, two female subjects also had the worst outcome measures and showed a decrease in speech recognition ability one year after implantation. The two male subjects improved in their post-test clinical measures, but not enough to be considered successful (less than $20 \%$ improvement). The average performance of the six females was greater (i.e., a $32 \%$ improvement) than the average performance of the two males (i.e., a $2.5 \%$ improvement), which appears to be a significant difference; however, with only eight subjects in the study, associations between gender and clinical outcome measures could not be made with certainty.

\subsection{Etiology of Hearing Loss}

Etiology may play an important role in the influence of achieving a good outcome. For example, if the lesion is localized in the cochlea not in the nerve. However, This study was unable to conclude that any particular etiology can be associated with good or poor speech recognition outcomes due to a small sample size, and the fact that the subjects' underlying etiology either varied considerably or was unknown.

\subsection{Age of Implantation \& Duration of Deafeness}

Age may possibly be an independent significant factor when speech recognition performance with an implant declines with duration of deafness. In this study, subjects were implanted at variable ages (range $=$ four to forty-one 
years). For the successful subjects (speech recognition outcomes improving $20 \%$ or more), the mean age was around sixteen years. The mean age for subjects with less than a $20 \%$ improvement, or poor clinical outcomes, was twenty-four years. Based on these findings, age of implantation (or perhaps the duration of deafness in this case) may have affected the subject's speech recognition ability. However, the number of subjects in this study may not be sufficient to support age of implantation as a factor that impacts speech recognition outcome. Furthermore, although the mean age is higher for the group with poor speech recognition outcomes, there are subjects who improved less than $20 \%$ and that were implanted at a younger age than those in the successful group. Therefore, based on the limited retrospective data for the limited number of subjects, there does not appear to be a direct association between age of implantation for the prelingually deafened individual and speech recognition scores.

\subsection{Type of CI Device}

In this study, six of eight subjects opted for a Nucleus device; the remaining two chose Bionic. The type of speech processor varied among subjects. Specifically, subjects were implanted in either the right or left ear with one of the following devices: CII Bionic with Harmony speech processor (case 8), Nucleus 24 Contours with Freedom speech processor (case 4), Freedom Contour Advance (case 6 and case 7), Nucleus Cochlear Freedom Contour with a speech processor 2 (case 2), Bionics 90k (case 3), Nucleus 512 (case 5), Nucleus Cochlear Freedom Contour (case 1). Case 8 was deemed as having unsuccessful speech recognition scores, whereas case 3 was deemed as having successful speech recognition scores. Both these subjects used a Bionics device; however, the successful subject used a Bionics 90k instead of a harmony speech processor. The difference in speech processors may have played a part role in the degree of success regarding the speech recognition outcome. However, as there were only two subjects that used a Bionics device, this could not be regarded as an affecting factor. Looking at the data regarding other devices, it can be seen that both subjects who used a Freedom Contour Advance CI (i.e., case 7 and case 6) were also classified as having unsuccessful speech recognition outcomes. Two subjects that were implanted with a Nucleus Cochlear Freedom Contour CI (i.e., cases 2 and case 1) were classified as having a successful speech recognition outcome. It was also observed that three out of the four subjects who were deemed to have successful speech recognition scores were implanted with relatively newer CI (e.g., Nucleus Cochlear Freedom Contour and Bionics 90k).

Successful and unsuccessful speech recognition scores post-implantation were achieved by both Nucleus and Bionic users; therefore, based on the information available from the retrospective data, the type of device implanted in a prelingual individual with bilateral sensorineural hearing loss does not appear to be associated with either a poor or successful outcome. More research is needed in order to determine if newer CIs increase speech recognition outcomes. More data would be required to determine if there is a link between the type of $\mathrm{CI}$ and speech recognition outcomes for this special CI population (prelingual CI users) instead of a general CI population (postlingual CI users).

\subsection{Oral Communication}

Oral, speech reading, and/or sign language were the modes of communication used among the subjects in the current study. Table 1 shows the variability of this factor among subjects; the majority of subjects used combinations of communication type that were different from each other. Oral and speech reading was a common factor between case 2 (relatively the second most successful subject) and case 8 (relatively the least successful subject). For these two cases, the influence of communication mode on speech recognition outcome could not be determined. Due to the limited available data and unknown communication types for two subjects in this study, associations between type of communication and speech recognition outcome could not be determined with statistical significance in the current study.

On the other hand, it was also observed from the findings that $75 \%$ of subjects (i.e., three out of four: Cases 1-4) used oral communication as their main communication modality and had successful speech perception outcomes post cochlear implantation. In contrast, only $50 \%$ of subjects (i.e., two out of four: Cases 5-8), deemed to have poor speech perception outcomes post cochlear implantation, used oral communication. Even within poor outcome group (Cases 5-8), the two who were noted to also use oral communication scored better than other two who were not noted. For example, case 4 used oral communication, speech recognition, and sign; she was determined to have relatively successful speech recognition scores. There were no other subjects for comparison who used a combination of oral communication, speech recognition, and sign. Furthermore, case 6 used speech reading and writing for communication; his post implantation speech recognition score was relatively unsuccessful. There were no other subjects for comparison who used the same modes of communication. Case 7, relatively unsuccessful, used oral communication and sign. The subject with the most successful speech recognition outcome, case 1 , used only oral communication. She was the only known subject to use oral speech for communication exclusively among subjects whose information of communication mode was available. It appears that either the use of oral communication only (based on the outcome measure for case 1) or use of combination with oral communication together (based on the outcome measure for other cases as described above).

\subsection{Compliance}

It was noted that $50 \%$ of "successful" subjects (i.e., those 
subjects that showed improved speech perception outcomes post implantation) had attended all medical appointments. Two subjects with successful outcomes, case 3 and case 1, were reported to have attended all scheduled medical appointments. Data for compliance (measured by the number of times the subject attended a scheduled medical appointment) was not available for the remainder of the subjects. It was speculated that the compliance demonstrated by the two subjects, who were reported to have attended all scheduled medical appointments, may have contributed to their successful speech recognition outcomes $(100 \%$ improvement on the HINT for case 1 and $30 \%$ improvement on the CID for case 3). However, because of the lack of similar information for the other subjects, there is not enough evidence from this case series study to statistically conclude that attendance to medical appointments may be associated with successful post-implantation performance.

\subsection{Family and Environment}

$75 \%$ of subjects that received improved speech perception outcomes appeared to have positive family and environment interactions. For example, case 1 reported that she talked on the telephone with her relatives. One may assume that case 1's family supported her and was involved in her life; they took the time and effort to interact with her over the phone, even though these conversations may have been difficult due to her speech and hearing difficulties. Case 2 also used the phone, as well as listened to music. These activities may suggest that the subject actively explored environments to maximize her engagement and CI performance. The youngest subject, case 4 , had been provided with many hours of help and assistance using her CI, suggesting that support may be an important environmental factor in successful speech recognition outcomes. Although not known for sure, the researchers believe that supportive relationships and environments are integral to improving speech perception outcomes for all CI users.

\subsection{Use of CI Device}

Use of CI use was not originally included as a factor in this study as there might be not enough data available for comparison; however, the researchers believe that the frequency and duration of $\mathrm{CI}$ use deserves mention as a possible influence on the clinical outcomes. Case 8 , for example, had reported limited use of the device as well as poor speech recognition outcomes pre and post-implantation. Case 6 did not wear his device during the day due to his work environment, and he also had unsuccessful clinical measures post-implantation. It may be likely that the speech recognition skills of case 8 and case 6 were not progressing due to their limited use of the CI device; therefore, a lack of or reduction in CI use has been speculated to be associated with poor speech recognition abilities. Furthermore, case 2 and case 1 used listening devices frequently (i.e. every day), and they demonstrated relatively successful outcome measures for speech recognition. Case 2 wore hearing aids starting at age two years and five months until age twenty-four when she no longer benefitted from wearing them. Approximately five years later, she received a CI; she reported satisfaction with her device, and that she was beginning to rely less on speech/lip reading. Case 1 used her $\mathrm{CI}$ all waking hours, and it was reported that she has been performing well with the device.

\section{Discussion}

In the section above, the cases have been presented uniquely based on the eight factors. Therefore, this section will discuss these eight factors, which are distinct among regular case (series) report. This will be addressed in the following section in order to further explore possible associations of these factors to the clinical outcome measures.

\subsection{Gender}

Not many studies have been able to statistically associate gender with predictions of speech recognition scores post-implantation. A retrospective study of adult CI users (varying in pre-, peri- and post-lingual deafness) by Bodmer et al. [8] found that neither poor nor excellent speech discrimination scores post-CI correlated statistically with gender (although 39\% of excellent performers were male, $61 \%$ were female). Another retrospective study of post-lingually deafened adults by Green \& colleagues showed higher speech recognition scores (measured in correct number of keywords identified out of 100 total words) for females (mean score of 57.6) than for males (mean score of 48.9) on the Bamford-Kowal-Bench (BKB) sentence test, but statistically found no significant difference between the two genders[9]. Zhu and colleagues studied Mandarin Chinese speech recognition by CI users (pre and postlingually deaf children) and found better performance for females ( $84 \%$ correct on disyllable test and $85 \%$ correct on sentences) than for males ( $78 \%$ correct on disyllable test and $80 \%$ correct on sentences)[10]. Finally, a study by Lenarz and colleagues examined the effect of gender on the hearing performance for postlingual adult CI subjects[11]. Their study revealed that men scored slightly higher than women in all of the follow-up sessions for the Hochmair-Schulz-Moser (HSM) Test in quiet, a contradictory finding to this author's current study with prelingual individuals with CIs. It is clear that more research regarding gender and speech recognition for the prelingual individual is required, as studies with postlingual subjects may yield different results. Based on the studies above, although not conclusive, it appears that female prelingual CI users tend to perform better than male prelingual CI users. The results of these former studies are consistent with our findings, and although the sample size in our study is small, it is interesting to note that $100 \%(4 / 4)$ of the subjects in the 
"good performance group" are female, while in the "poor performance group," only $50 \%(2 / 4)$ of the participants are female.

\subsection{Etiology of Hearing Loss}

van Dijk and colleagues indicated that research was showing no significant relationship between etiology of hearing loss and speech understanding for post-lingually deafened CI users[12]. More recently, in terms of genetics, Dalamon et al. found no differences in prelingually deaf CI users' speech perception scores, whether their hearing loss was caused by non-syndromic genetic factors or by unknown etiology[13].

Similar to the research above, this study is unable to conclude that any particular etiology can be associated with good or poor speech recognition outcomes. This is due to our small sample size, and that the subjects' underlying etiology either varied considerably or was unknown.

Research has only been able to conclude that prelingual hearing loss caused by meningitis, especially if the disease causes ossification of the cochlea, may lead to poorer outcomes[14], while in post-lingual deafness, although also caused by osteosclerosis, the outcomes can be better[12]

Other studies have indicated that individuals with congenital and prelingual loss of hearing show poorer outcomes than those with a progressive or postlingual $\operatorname{loss}[15]$.

\subsection{Age of Implantation \& Duration of Deafness}

Age could be an independent significant factor when speech recognition performance with an implant declines with duration of deafness[16]. According to Tyler \& Summerfield, the longer the period of profound deafness before implantation in post-lingual individuals, the poorer the outcome in Speech Recognition Testing (SRT)[16]. A longer duration of deafness and older age may be associated with reductions in the responsiveness of the nervous system and in the potential for relearning[16].

Studies like that by Arisi et al. also suggest that speech recognition performance in adolescent $\mathrm{CI}$ recipients is affected by age at implantation and length of deafness. Prelingually deafened adolescents $(\mathrm{N}=45)$ with a mean age at implantation of 13.4 years (range 11-18 years) participated in their study[17]. Arisi et al. found that age at implantation, duration of deafness, and preoperative hearing threshold affect speech recognition outcomes[18].

Cosetti \& Waltzman presented many research studies that support improved auditory and linguistic outcomes in children who are implanted before twelve months of age[7]. For instance, in 2010, Tajudeen et al. found that CI recipients less than one year old $(\mathrm{N}=35)$ may have a significant advantage in areas of speech recognition compared with later-implanted groups[15]. Similarly, Dettman \& colleagues examined receptive and expressive language of nineteen children implanted before twelve months of age using the
Rosetti Infant-Toddler Language Scale (RI-TLS)[19]. Results indicated language scores comparable to typically hearing children, and better scores than children implanted between twelve and twenty-four months[19]. Niparko et al. showed similar results for children implanted before eighteen months of age[20].

The studies above suggest that age at implantation and length of deafness have a clear impact on CI performance in children, prelingual adolescents, and post-lingual adults[7]. Age of implantation is linked to duration of deafness in the prelingually deaf population: all prelingually deaf individuals become deaf before learning language; therefore, they would have similar deafness durations. According to the literature discussed above, a shorter duration of deafness can be associated with better speech recognition outcomes post-implantation. Age at implantation was found to affect speech recognition outcomes in prelingual adolescents[17].

In our study, the average age in the "good user group" is younger than the "poor user group" (16 vs. 24 years old), which is consistent with the aforementioned studies.

\subsection{Type of CI Device}

At the time of this study, there were three major CI manufactures: Cochlear Corporation, MedEI Corporation, and Advanced Bionics Corporation. Although the success of a $\mathrm{CI}$ is dependent on the individual person, both the device type and the sound processing strategy used are variables that can influence the success of cochlear implementation.

Santerelli et al. tested a group of seventeen prelingually deaf children who had received the Nucleus 24 CI[21]. All of these children had been implanted before the age of ten and had either received the Sprint or the Esprit 3G sound processor. Both of these sound processors had a limited dynamic range input. Spahr et al. had found that differences in speech recognition in a group of postlingually deaf CI users were mainly related to differences in the input dynamic ranges of the sound processor[22]. Santerelli et al. found that when these children were given a sound processor with an increased input dynamic range (i.e., Freedom)[21], and their speech perception scores were superior to their scores with the Esprit $3 \mathrm{G}$ or the Sprint. It is thought that a sound processor with an increased input dynamic range allows for more phonemic discrimination.

Technical improvements in CIs can be another factor that contributes to a subject's success with speech perception. Digisonic Convex and Digisonic SP are two recent, widely used Neurelec CI devices that were released in 1999 and in 2004 respectively. These devices were compared to each other by looking at speech recognition scores in one-hundred postlingually deaf CI adults who had either been implanted with the Digisonic Convex or the Digisonic SP. Speech perception scores were obtained one year after implantation; subjects who had received the Digisonic SP (the newer CI devices) had better speech recognition scores overall than those with the Digisonic Convex device, and their skills continued to improve over a longer period of time[23]. It was 
found by Lenarz and colleagues that post lingual subjects who had received more recent electrode designs and speech processing strategies (i.e., developed between 2002 and 2008) had better speech perception in noisy surroundings than those subjects who had the earlier technology[24].

CI technology continues to improve. New devices include the totally implantable CI (TIKI), in which there are no external components. This allows people to utilize their CI in situations where they have previously been unable to due to the external component. Other technologies that have been seen to improve hearing outcomes are improvements in speech processing strategies, microphone technologies, and electrode placement[18].

In the current study, it was speculated that the sound processor of the CI device may be a factor that leads to either a poor or successful outcome. Spahr et al. found that greater dynamic range input of the sound processor lead to better speech recognition[22]. Lazard et.al. determined that the subjects who had been implanted with the Digisonic SP device (a device where all twenty electrodes could be activated on the electrode array) had better identification scores than those subjects who used the Digisonic Convex device (a device where only fifteen electrodes could be activated) [23]. Lenarz et al. preformed a study and determined that adult CI subjects implanted with the most recent electrode designs and speech processors had better speech perception in noisy environments than those subjects who used an older/less recent speech processor[24].

\subsection{Method(s) of Communication}

Previous research has shown that children exposed to oral communication before implantation have better speech perception outcomes post implantation[7]. Taitelbaum-Swead et al. also showed that children with congenital hearing loss who used oral communication in addition to their CI's showed better speech recognition outcomes than children (CI users) who used sign language[15]. Similarly, Kaplan et al. (2003)[25] found that prelingually deaf adults implanted in adulthood who came from "a strong auditory-oral background" performed better on speech recognition tasks. Kirk and colleagues (2002)[26] found that children who used oral communication (exclusively) had higher word recognition scores than children who used total communication.

The subject with the most successful speech recognition outcome in the current study, case 1, used only oral communication. Based on the outcome measure for case 1 , it appears that the use of oral communication only may yield relatively successful speech recognition outcomes. The successful outcome of case 1 is consistent with the findings of those described above[ $7,15,25,26]$.

\subsection{Attendance to Medical Appointments (Compliance)}

A study by Fitzpatrick \& Schramm identified subject motivation, perseverance, and commitment to rehabilitation as being factors that impact subject communication and the length of the rehabilitation period required for intelligible speech production in adults with prelingual deafness[6]. Furthermore, accuracy of speech recognition for postlingually deafened adults with implants has been thought to relate to the subject's preoperative motivation to learn to use the implant[16]. Based on our data, we propose that a subject who is highly motivated to use his/her implant may be more likely to follow the rehabilitation plan and use his/her implant in a variety of situations to communicate with others. We speculate that attendance to medical appointments can be associated with overall motivation and compliance. Our findings support those from Fitzpatrick \& Schramm and Tyler \& Summerfield[6, 16]. Motivation can come from external factors (e.g. family and friends) or internal factors (e.g. the desire to be understood by others).

\subsection{Family and Environment}

Positive family involvement and environment may improve speech recognition measures for prelingual CI users. Consistent with our speculations that family and environment may influence outcome measures, Holt et al. found that for prelingually deafened children implanted between 0.7 and 6.8 years of age, family environment influences CI outcomes in language development[27]. We believe that without sufficient family involvement and supportive environments, subjects may abandon their efforts to succeed with their CIs; thus, their speech recognition abilities may not be maximized. However, as Holt et al. stated, "family environment can be modified and enhanced by therapy or education," [27], this is an important idea for clinicians to consider.

\subsection{Use of CI Device}

It may be likely that the speech recognition skills of case 8 and case 6 were not progressing due to their limited use of their CI device. This is consistent with a previous study: a lack of or reduction in CI use has been speculated to be associated with poor speech recognition abilities[28]. According to Fu \& Galvin, CI recipients may have difficulty adjusting to the electrically stimulated speech patterns; however, through passive, daily-listening experiences, most implant recipients will adapt at least partially to the new patterns of electrically stimulated speech[29]. The research by $\mathrm{Fu} \&$ Galvin is consistent with our speculation that the frequency and duration of $\mathrm{CI}$ use may influence the clinical outcome measures for speech recognition[29].

\section{Conclusions}

There have been studies regarding the speech recognition outcomes of post-lingual[12] and pre-lingual CI users[5]. However, the study of pre-implantation and post-implantation factors that influence the clinical measures 
in the prelingual subject is unique; although researchers like Cosetti \& Waltzman have considered pre-operative factors in their research, the majority of their studies targeted post-operative variables affecting CI performance[7]. Like many other studies focusing on the outcomes of prelingual users that were unable to make conclusions based on statistical significance, our finding from this investigation is also not statistically conclusive. However, although not conclusive, it appears that some factors, such as patient compliance, oral communication, family environment, and/or the frequency using the CI device, may contribute to positive speech recognition outcomes. Therefore, prelingual individuals should not be excluded as candidates for $\mathrm{CI}$ at this time. In addition, based on the findings in the cases reported, evaluation of these findings, and discussion in this study, it is clear that additional and further more in-depth research on these eight factors and four potential positive factors are warranted.

\section{Acknowledgements}

Part of this work was supported by funding from Social Sciences and Humanities Research Council (E.H.), grants from Glenrose Rehabilitation Hospital Foundation (M.Z.), Research Matching Funds from the University of Alberta (M.Z.), and Queen Elizabeth II Graduate Scholarship. The authors wish to thank Marla Baum for her great contributions to the project, Dr. Douglas Gross for his insightful discussions and review of this study, Dr. Bill Hodgetts for his input, and Krista Greidanus for her assistance in data collection.

\section{REFERENCES}

[1] M. Manrique, F.J. Cervera-Paz, A. Huarte and M. Molina, "Advantages of cochlear implantation in prelingual deaf children before 2 years of age when compared with later implantation," Laryngoscope, Vol. 114, No. 8, pp. 1462-9, 2004.

[2] R.F. Holt and M.A. Svirsky, "An exploratory look at pediatric cochlear implantation: Is earliest always best?," Ear Hear, Vol. 29, No. 4, pp. 492-511, 2008.

[3] N. Tye-Murray, Foundations of aural rehabilitation: Children, adults, and their family members, Clifton Park, NY, Delmar Cengage Learning, 2009.

[4] B.J. Gantz, R.S. Tyler, G.G. Woodworth, N. Tye-Murray and H. Fryauf-Bertschy, "Results of multichannel cochlear implants in congenital and acquired prelingual deafness in children: Five-year follow-up," Am J Otol, Vol. 15 Suppl 2, No. pp. 1-7, 1994.

[5] T. Shpak, L. Koren, N. Tzach, T. Most and M. Luntz, "Perception of speech by prelingual pre-adolescent and adolescent cochlear implant users," Int J Audiol, Vol. 48, No. 11, pp. 775-83, 2009.
[6] E.M. Fitzpatrick and D. Schramm, "Clinicians' perceptions of cochlear implant benefits in adults with prelingual deafness," Journal of Speech-Language Pathology and Audiology, Vol. 30, No. 3, pp. 192-197, 2006.

[7] M.K. Cosetti and S.B. Waltzman, "Outcomes in cochlear implantation: Variables affecting performance in adults and children," Otolaryngol Clin North Am, Vol. 45, No. 1, pp. 155-71, 2012.

[8] D. Bodmer, D.B. Shipp, J.M. Ostroff, A.H. Ng, S. Stewart, J.M. Chen, and J.M. Nedzelski, "A comparison of postcochlear implantation speech scores in an adult population," Laryngoscope, Vol. 117, No. 8, pp. 1408-11, 2007.

[9] K.M. Green, Y. Bhatt, D.J. Mawman, M.P. O'Driscoll, S.R. Saeed, R.T. Ramsden, and M.W. Green, "Predictors of audiological outcome following cochlear implantation in adults," Cochlear Implants Int, Vol. 8, No. 1, pp. 1-11, 2007.

[10] M. Zhu, Q.J. Fu, J.J. Galvin, 3rd, Y. Jiang, J. Xu, C. Xu, D. Tao, and B. Chen, "Mandarin chinese speech recognition by pediatric cochlear implant users," Int $J$ Pediatr Otorhinolaryngol, Vol. 75, No. 6, pp. 793-800, 2011.

[11] M. Lenarz, H. Sonmez, G. Joseph, A. Buchner and T. Lenarz, "Effect of gender on the hearing performance of adult cochlear implant patients," Laryngoscope, Vol. 122, No. 5, pp. 1126-9, 2012.

[12] J.E. van Dijk, A.F. van Olphen, M.C. Langereis, L.H. Mens, J.P. Brokx and G.F. Smoorenburg, "Predictors of cochlear implant performance," Audiology, Vol. 38, No. 2, pp. 109-16, 1999.

[13] V. Dalamon, V. Lotersztein, M. Lipovsek, A. Beheran, M.E. Mondino, F. Diamante, N. Pallares, V. Diamante, and A.B. Elgoyhen, "Performance of speech perception after cochlear implantation in dfnb1 patients," Acta Otolaryngol, Vol. 129, No. 4, pp. 395-8, 2009.

[14] H.K. El-Kashlan, C. Ashbaugh, T. Zwolan and S.A. Telian, "Cochlear implantation in prelingually deaf children with ossified cochleae," Otol Neurotol, Vol. 24, No. 4, pp. 596-600, 2003.

[15] R. Taitelbaum-Swead, L. Kishon-Rabin, R. Kaplan-Neeman, C. Muchnik, J. Kronenberg and M. Hildesheimer, "Speech perception of children using nucleus, clarion or med-el cochlear implants," Int J Pediatr Otorhinolaryngol, Vol. 69, No. 12, pp. 1675-83, 2005.

[16] R.S. Tyler and A.Q. Summerfield, "Cochlear implantation: Relationships with research on auditory deprivation and acclimatization," Ear Hear, Vol. 17, No. 3 Suppl, pp. 38S-50S, 1996.

[17] E. Arisi, S. Forti, D. Pagani, L. Todini, S. Torretta, U. Ambrosetti, and L. Pignataro, "Cochlear implantation in adolescents with prelinguistic deafness," Otolaryngol Head Neck Surg, Vol. 142, No. 6, pp. 804-8, 2010.

[18] R.J. Briggs, "Future technology in cochlear implants: Assessing the benefit," Cochlear Implants Int, Vol. 12 Suppl 1, No. pp. S22-5, 2011.

[19] S.J. Dettman, D. Pinder, R.J. Briggs, R.C. Dowell and J.R. Leigh, "Communication development in children who receive the cochlear implant younger than 12 months: Risks versus benefits," Ear Hear, Vol. 28, No. 2 Suppl, pp. 11S-18S, 2007. 
[20] J.K. Niparko, E.A. Tobey, D.J. Thal, L.S. Eisenberg, N.Y. Wang, A.L. Quittner, and N.E. Fink, "Spoken language development in children following cochlear implantation," Jama, Vol. 303, No. 15, pp. 1498-506, 2010.

[21] R. Santarelli, V. Magnavita, R. De Filippi, L. Ventura, E. Genovese and E. Arslan, "Comparison of speech perception performance between sprint/esprit $3 \mathrm{~g}$ and freedom processors in children implanted with nucleus cochlear implants," Otol Neurotol, Vol. 30, No. 3, pp. 304-12, 2009.

[22] A.J. Spahr, M.F. Dorman and L.H. Loiselle, "Performance of patients using different cochlear implant systems: Effects of input dynamic range," Ear Hear, Vol. 28, No. 2, pp. 260-75, 2007.

[23] D.S. Lazard, P. Bordure, G. Lina-Granade, J. Magnan, R. Meller, B. Meyer, E. Radafy, P.E. Roux, D. Gnansia, V. Pean, and E. Truy, "Speech perception performance for 100 post-lingually deaf adults fitted with neurelec cochlear implants: Comparison between digisonic(r) convex and digisonic(r) sp devices after a 1-year follow-up," Acta Otolaryngol, Vol. 130, No. 11, pp. 1267-73, 2010.

[24] M. Lenarz, G. Joseph, H. Sonmez, A. Buchner and T. Lenarz, "Effect of technological advances on cochlear implant performance in adults," Laryngoscope, Vol. 121, No. 12, pp.
2634-40, 2011

[25] D.M. Kaplan, D.B. Shipp, J.M. Chen, A.H. Ng and J.M. Nedzelski, "Early-deafened adult cochlear implant users: Assessment of outcomes," J Otolaryngol, Vol. 32, No. 4, pp. 245-9, 2003.

[26] K. Kirk, R. Miyamoto, E. Ying, A. Perdew and H. Zuganelis, "Cochlear implantation in young children: Effects of age at implantation and communication mode," Volta Review, Vol. 102, No. 4, pp. 127-144, 2002.

[27] R.F. Holt, J. Beer, W.G. Kronenberger, D.B. Pisoni and K. Lalonde, "Contribution of family environment to pediatric cochlear implant users' speech and language outcomes: Some preliminary findings," J Speech Lang Hear Res, Vol. 55, No. 3, pp. 848-64, 2012.

[28] H. Fryauf-Bertschy, R.S. Tyler, D.M. Kelsay, B.J. Gantz and G.G. Woodworth, "Cochlear implant use by prelingually deafened children: The influences of age at implant and length of device use," J Speech Lang Hear Res, Vol. 40, No. 1, pp. 183-99, 1997.

[29] Q.J. Fu and J.J. Galvin, 3rd, "Perceptual learning and auditory training in cochlear implant recipients," Trends Amplif, Vol. 11, No. 3, pp. 193-205, 2007. 